\title{
Cognitive Profiles of Patients with Mild Cognitive Impairment or Dementia in Alzheimer's or Parkinson's Disease
}

\author{
Helmut Hildebrandt ${ }^{a}$, $b$ Frauke Fink ${ }^{b, c}$ Andreas Kastrup ${ }^{a}$ \\ Michael Haupts $^{b}$ Paul Eling ${ }^{d}$ \\ ${ }^{a}$ Department of Neurology, Klinikum Bremen-Ost, Bremen, ${ }^{b}$ Institute of Psychology, \\ University of Oldenburg, Oldenburg, and 'Department of Neurology, Augustahospital \\ Anholt, Isselburg, Germany; ${ }^{\mathrm{d}}$ Donders Institute for Brain, Cognition and Behavior, \\ Radboud University of Nijmegen, Nijmegen, The Netherlands
}

\author{
Key Words \\ Parkinson's disease · Alzheimer's disease - Mild cognitive impairment · Dementia · \\ Familiarity $\cdot$ Recollection $\cdot$ Mood $\cdot$ Disease progression
}

\begin{abstract}
Background: Alzheimer's disease (AD) and Parkinson's disease (PD) are associated with severe cognitive decline, but it is still unclear to what extent they become functionally more similar over time. Methods: We compared amnestic mild cognitively impaired ( $a M C I ; n=29)$ patients to mild cognitively impaired (MCI) PD patients $(n=25)$, and patients with $A D(n=34)$ to patients with PD dementia (PDD; $n=15$ ) with respect to cognitive functioning and mood. $\boldsymbol{R e}$ sults: aMCI patients were impaired in episodic memory, while MCI PD patients showed deficits in visuoconstruction and attention. $A D$ and PDD patients showed comparable deficits on tests for language, attention and visuoconstruction. However, unlike PDD patients but similar to aMCI patients, AD patients showed a characteristic memory impairment, especially commission errors on recognition tasks, whereas PDD patients scored higher on the depressive mood questionnaire. Conclusions: In advanced stages of both diseases, the pattern of functional deficits associated with parietal and temporal lobe functions (attention, visuoconstruction and language) is similar. However, specific differences, already present in the early stage (recognition errors in $A D$, associated with mediobasal temporal lobe functioning, and depressed mood in PDD, associated with non-motor basal ganglia loops), are also observed in the late stage.




\section{Introduction}

Alzheimer's disease (AD) and Parkinson's disease (PD) are the most frequent neurodegenerative disorders. AD is characterized by a progressive cognitive decline. However, also the majority of PD patients develops dementia in the course of the disease, and a considerable number of patients already shows cognitive deficits in the early stage, which are severe enough to be diagnosed as dementia [1-5].

Several neuropsychological studies have investigated whether the pattern of cognitive deficits in AD can be distinguished from that in PD [6-10]. There is now a fair consensus that $\mathrm{AD}$ is characterized by a severe decline in episodic memory, whereas PD is typically associated with executive and visuoconstructive deficits in combination with psychomotor slowing $[11,12]$. Nonetheless, these conclusions are mainly based on an indirect comparison of patients suffering from each of these disorders with healthy control groups, and the number of studies directly comparing these two patient groups is rather small [13-16].

From a neuropathological point of view and against the background of disease-specific pathogenetic processes, it can be argued that $\mathrm{AD}$ and $\mathrm{PD}$ patients should show different patterns of cognitive deficits. For instance, according to the Braak stages in AD, the development of neurofibrillary tangles starts in the entorhinal cortex, extends into the hippocampus and then spreads to secondary association cortices [17]. In PD, neurodegeneration starts in the brain stem, extends into the midbrain, affecting the substantia nigra, and subsequently into cortical areas [17]. These differences in neuropathological changes should lead to an increasing difference in cognitive impairments during the progression of the two diseases. However, more recent neuropathological studies have challenged this view $[18,19]$. In fact, several animal studies have recently shown that Lewy-related pathology may stimulate the development of beta-amyloid plaques and vice versa [17, 20]. Furthermore, postmortem neuropathological studies have shown that many PD patients fulfill the criteria of AD on neuropathological grounds [21].

These findings cast some doubt on the notion that the two diseases are characterized by different patterns of cognitive deficits. The strongest evidence for an interaction between accumulation of alpha-synuclein in Lewy bodies and beta-amyloid in plaques stems from animal research $[17,20]$ because in humans this interaction cannot be studied, at least not until valid biomarkers for the progressive development of Lewy bodies and beta-amyloid plaques are available. The question whether these neuropathological processes interact also in patients with $\mathrm{AD}$ or $\mathrm{PD}$ can be addressed, to some extent, by looking at behavioral data. More specifically, we can study whether the pattern of deficits becomes increasingly similar in the course of the diseases. Yet, the number of studies directly comparing patients with AD and PD in the course of their illness is quite small and in some of these studies the MiniMental State Examination (MMSE) is used, which, however, is not very sensitive $[9,14,16$, 21]. We have shown that on a recognition task, AD patients typically made commission errors (false positives). This specific deficit correlated with biomarkers like beta-amyloid and total tau [22,23], and this task seems to be sensitive to early cognitive problems in AD. In the current study, we extend our previous research by comparing patients with AD and PD, either in an early state of mild cognitive impairment (MCI) or in a state of dementia, specifically addressing the following questions: (1) Is the pattern of cognitive deficits in AD and PD similar in an early stage (i.e. of MCI) and in a severe state of cognitive decline? (2) Are commission errors characteristic for AD patients, both in an early and late phase of the disease? 
Table 1. Patients with AD and PDD

\begin{tabular}{|c|c|c|c|c|c|c|}
\hline & \multicolumn{3}{|c|}{ AD (females: 23, males: 11) } & \multicolumn{3}{|c|}{ PDD (females: 10, males: 5) } \\
\hline & mean & median & SD & mean & median & SD \\
\hline Age, years & 71.2 & 72.0 & 7.6 & 73.3 & 74.0 & 7.4 \\
\hline Education, years & 12.8 & 12.0 & 3.5 & 11.3 & 12.0 & 2.6 \\
\hline MMSE & 17.0 & 18.0 & 5.6 & 18.9 & 22.0 & 6.0 \\
\hline Tau protein, pg/ml & 767 & 531 & 578 & - & - & - \\
\hline Beta-amyloid, pg/ml & 357 & 357 & 121 & - & - & - \\
\hline Hoehn and Yahr stage & - & - & - & 3.3 & 3 & 0.6 \\
\hline Beck Depression Inventory* & 8.9 & 5.0 & 9.9 & 14.2 & 15.5 & 6.1 \\
\hline Rey Verbal Comprehension & 4.1 & 5.0 & 1.6 & 3.9 & 4.0 & 1.0 \\
\hline Mehrfachwahl-Wortschatz-Intelligenztest & 24.8 & 25.5 & 7.9 & 24.4 & 24.0 & 4.5 \\
\hline Boston Naming Test ${ }^{1}$ & 12.1 & 11.0 & 3.7 & 12.5 & 13.0 & 2.2 \\
\hline Semantic fluency ${ }^{1}$ & 10.6 & 10.5 & 5.9 & 10.9 & 9.0 & 6.1 \\
\hline LPS subtest 6 (phonological fluency) & 16.5 & 14.0 & 9.0 & 16.6 & 15.5 & 4.8 \\
\hline ZVT, s & 111.2 & 77.3 & 95.8 & 82.0 & 53.5 & 62.0 \\
\hline Digit Span forward & 5.8 & 6.0 & 1.9 & 6.5 & 6.5 & 2.6 \\
\hline Digit Span backward & 3.6 & 4.0 & 1.7 & 3.3 & 3.0 & 1.9 \\
\hline Copying ${ }^{1}$ & 8.0 & 8.0 & 2.3 & 7.8 & 8.0 & 2.1 \\
\hline Word list learning ${ }^{1, *}$ & 8.1 & 8.0 & 3.9 & 11.1 & 12.0 & 5.8 \\
\hline Word list delayed recall ${ }^{1}$ & 1.4 & 1.0 & 1.7 & 2.1 & 2.0 & 2.1 \\
\hline Word list intrusions $^{1}$ & 1.9 & 1.0 & 2.6 & 1.1 & 1.0 & 1.8 \\
\hline Word list savings $^{1}$ & 35.2 & 33.0 & 36.8 & 40.1 & 40.0 & 35.7 \\
\hline Visuoconstructive delayed recall ${ }^{1}$ & 1.9 & 1.0 & 2.4 & 2.7 & 3.0 & 2.9 \\
\hline Visuoconstructive savings $^{1}$ & 22.4 & 13.0 & 26.4 & 31.5 & 36.0 & 32.6 \\
\hline Word list discriminability ${ }^{1}$ & 73.8 & 75.0 & 14.1 & 83.7 & 85.0 & 13.2 \\
\hline Word list recognition: correct hits ${ }^{1}$ & 3.1 & 2.0 & 2.8 & 2.5 & 3.0 & 1.5 \\
\hline Word list recognition: correct rejections ${ }^{1, *}$ & 2.1 & 1.0 & 2.4 & 0.9 & 0.0 & 1.8 \\
\hline Visual recognition task: omissions & 4.4 & 3.0 & 3.6 & 5.3 & 5.0 & 3.6 \\
\hline Visual recognition task: commissions* & 5.3 & 4.5 & 4.1 & 2.5 & 1.0 & 3.1 \\
\hline
\end{tabular}

${ }^{1}$ CERAD-NP battery. $* \mathrm{p}<0.05$.

\section{Methods}

\section{Patients}

Twenty-nine patients with amnestic MCI (aMCI), 25 patients with PD and MCI but no dementia, 34 patients with probable AD and 15 patients with PD dementia (PDD) were included (tables 1, 2). Patients were invited to participate in this study after their admission to the neurological departments of the two hospitals involved. All diagnoses were based on the guidelines of the German Neurological Society, which are updated every 3-5 years to follow the international consensus processes (http://www.dgn.org/images/stories/dgn/ pdf/s3_leitlinie_demenzen.pdf). The diagnosis of AD was based on clinical examination, neuropsychological assessment and on a decreased beta-amyloid $(<375 \mathrm{pg} / \mathrm{ml})$ or increased total tau level ( $>448 \mathrm{pg} / \mathrm{ml}$ ) [24]. Disease severity of the PD patients was assessed using the Hoehn and Yahr scale [25]. For both groups (AD and PDD), a sum score below 25 in the MMSE was defined as cutoff score for diagnosing a dementia syndrome. aMCI patients scored $1.5 \mathrm{SD}$ or more below the standard population mean on the word list learning task (see below) and reported a decrease in memory function. MCI PD patients scored 1.5 SD or more below the mean on at least one of the neuropsychological tests applied. Patients with any other neurological or psychiatric disease unrelated to $\mathrm{MCI}, \mathrm{AD}$ and PD were excluded. Exclusion was 
Hildebrandt et al.: Cognitive Profiles of Patients with Mild Cognitive Impairment or

Dementia in Alzheimer's or Parkinson's Disease

Table 2. Patients with aMCI and MCI PD

\begin{tabular}{|c|c|c|c|c|c|c|c|c|c|}
\hline & \multicolumn{3}{|c|}{$\begin{array}{l}\text { Healthy controls } \\
\text { (females: } 10 \text {, males: } 10 \text { ) }\end{array}$} & \multicolumn{3}{|c|}{$\begin{array}{l}\text { aMCI } \\
\text { (females: 14, males: } 11 \text { ) }\end{array}$} & \multicolumn{3}{|c|}{$\begin{array}{l}\text { MCI PD } \\
\text { (females: 16, males: 13) }\end{array}$} \\
\hline & mean & median & SD & mean & median & SD & mean & median & SD \\
\hline Age, years & 68.8 & 74.0 & 14.9 & 69.7 & 71.0 & 9.1 & 71.1 & 73.0 & 7.9 \\
\hline Education, years & 13.3 & 12.0 & 2.5 & 12.9 & 12.5 & 2.8 & 12.4 & 12.0 & 2.3 \\
\hline MMSE & 28.8 & 29.0 & 0.5 & 26.2 & 26.0 & 2.5 & 27.8 & 28.0 & 1.4 \\
\hline Tau protein, pg/ml & - & - & - & 459 & 355 & 356 & - & - & - \\
\hline Beta-amyloid, pg/ml & - & - & - & 658.2 & 637 & 302.7 & - & - & - \\
\hline Hoehn and Yahr Scale & - & - & - & - & - & - & 3.4 & 3.5 & 0.5 \\
\hline Beck Depression Inventory & 5.4 & 5.3 & 1.3 & 10.1 & 9.0 & 6.8 & 9.6 & 8.0 & 6.8 \\
\hline Rey Verbal Comprehension & 1.00 & 1.00 & & 2.65 & 3.00 & 1.50 & 2.52 & 2.00 & 1.94 \\
\hline Mehrfachwahl-Wortschatz-Intelligenztest & 31.8 & 31.5 & 2.1 & 28.0 & 30.0 & 4.9 & 27.6 & 27.0 & 5.2 \\
\hline Boston Naming Test ${ }^{1}$ & 15.0 & 15.0 & 0.0 & 13.9 & 14.0 & 3.3 & 14.0 & 15.0 & 1.4 \\
\hline Semantic fluency ${ }^{1}$ & 18.8 & 19.5 & 6.7 & 13.2 & 13.0 & 3.2 & 15.0 & 16.0 & 5.7 \\
\hline LPS subtest 6 (phonological fluency) & 27.5 & 26.5 & 6.2 & 22.0 & 22.0 & 8.9 & 21.7 & 21.0 & 8.7 \\
\hline ZVT, s* & 34.3 & 36.0 & 10.8 & 45.9 & 34.0 & 25.7 & 83.9 & 70.0 & 53.5 \\
\hline Digit Span forward* & 9.3 & 9.5 & 1.0 & 6.4 & 6.0 & 1.9 & 8.1 & 8.0 & 2.3 \\
\hline Digit Span backward & 8.0 & 8.0 & 1.6 & 5.6 & 5.0 & 4.6 & 5.4 & 5.0 & 1.8 \\
\hline Copying $^{1, *}$ & 10.3 & 11.0 & 1.5 & 9.4 & 10.0 & 1.2 & 9.0 & 9.0 & 2.0 \\
\hline Word list learning ${ }^{1}$ & 22.3 & 21.0 & 4.0 & 13.6 & 15.0 & 3.8 & 16.6 & 17.0 & 5.0 \\
\hline Word list delayed recall ${ }^{1, *}$ & 6.3 & 5.5 & 1.9 & 3.5 & 3.0 & 2.0 & 5.5 & 5.0 & 1.4 \\
\hline Word list intrusions ${ }^{1}$ & 0.8 & 1.0 & 0.5 & 0.9 & 0.0 & 1.2 & 0.9 & 1.0 & 1.7 \\
\hline Word list savings ${ }^{1, *}$ & 73.8 & 74.5 & 16.8 & 63.6 & 67.0 & 32.9 & 82.8 & 84.0 & 16.2 \\
\hline Visuoconstructive delayed recall $^{1}$ & 9.5 & 9.5 & 1.7 & 5.1 & 5.0 & 2.7 & 6.8 & 7.0 & 3.2 \\
\hline Visuoconstructive savings $^{1}$ & 93.3 & 100.0 & 13.5 & 53.6 & 56.0 & 27.4 & 73.6 & 75.0 & 29.5 \\
\hline Word list discriminability ${ }^{1}$ & 97.5 & 100.0 & 5.0 & 88.6 & 90.0 & 9.6 & 94.8 & 95.0 & 7.0 \\
\hline Word list recognition: correct hits ${ }^{1}$ & 0.5 & 0.0 & 1.0 & 1.4 & 1.0 & 1.4 & 0.9 & 0.0 & 1.4 \\
\hline Word list recognition: correct rejections ${ }^{1}$ & 0.5 & 0.0 & 1.0 & 0.8 & 0.0 & 1.0 & 0.2 & 0.0 & 0.5 \\
\hline Visual recognition task: omissions & 0.5 & 0.5 & 0.6 & 2.0 & 1.0 & 1.7 & 1.5 & 1.0 & 1.7 \\
\hline Visual recognition task: commissions* & 0.5 & 0.5 & 0.6 & 2.8 & 1.0 & 3.2 & 0.8 & 0.0 & 1.0 \\
\hline
\end{tabular}

${ }^{1}$ CERAD-NP battery. ${ }^{*} \mathrm{p}<0.05$ comparing aMCI with MCI PD patients.

based on the report of the patients, serological screening and on inspection of CCT or MRI scans. Twenty healthy individuals were recruited on an informal basis in our clinic and through an announcement at a location where elderly people meet. The project was approved by the Ethics Committee of the University of Oldenburg, Germany, and all patients gave their informed consent.

\section{Neuropsychological Investigation}

All participants (patients and healthy controls) underwent a neuropsychological examination, including the Consortium to Establish a Registry for Alzheimer's Disease (CERAD) test [26] series, composed of the MMSE, a 15-item short version of the Boston Naming Test, semantic word fluency for the animal category $(1 \mathrm{~min}$ ), word list learning (reading aloud of 10 words with the instruction to memorize the items, three trials), delayed recall of the word list (after the copying task), recognition (10 targets from the word list and 10 distractor words, directly after delayed recall), figure copying and delayed figure recall. We also used the letter fluency task from the Leistungsprüfsystem (LPS) 50+ Test [27], the German version of the FAS test. Further, we used the Zahlenverbindungstest (ZVT) [28], which is similar to the Trail Making Test, Version A. We used the Digit Span forward and backward from the German version of the Wechsler Memory Scale (revised) [29] to investigate verbal working memory 
performance. The Mehrfachwahl-Wortschatz-Intelligenztest (multiple choice vocabulary intelligence test [30]) was taken as a measure for the ability to discriminate between words and pseudowords. A German version of the Rey Verbal Comprehension task was used to test sentence comprehension and for constructing geometrical figures. The Beck Depression Inventory [31] was used to investigate mood. Neuropsychological examination was performed by an experienced and certified neuropsychologist.

\section{Investigation of Validity in Recognition}

In previous studies, we have successfully used a specially designed picture recognition task [22, 32]. Participants were asked to name 16 pictures and were not informed that they would be asked to recognize these later. After $15 \mathrm{~min}, 24$ recognition trials were given, each trial now encompassing 3 different pictures stemming from the same category, e.g. 3 insects, to increase discrimination difficulty. In 8 of the recognition trials, no picture was present that had been shown before, on 16 trials one of the three pictures had been shown before. The participants had to decide whether one of the three pictures had been shown before and, if so, which one. Misses were counted as omissions, false positive as commissions.

\section{Neurological Investigation}

All patients received a standard diagnostic examination, including medical history, physical and neurological examination, laboratory testing, brain imaging and electroencephalography. Blood sample analysis included determination of blood count, erythrocyte sedimentation rate, electrolytes, creatine, urea, transaminases, blood glucose, TSH and C-reactive protein. A lumbar puncture was performed by a trained neurologist in each aMCI and probable AD patient.

\section{Statistical Analysis}

For non-parametric testing, the Mann-Whitney U test was used to compare the patient groups. Since we anticipated that the healthy controls would perform better than any of the patient groups, analyses comparing healthy controls with patients were not performed.

In a first analysis, aMCI patients were compared to MCI PD patients irrespective of the MMSE score. In a second step, we matched the two groups using the MMSE score by restricting the range of MMSE scores, increasing the lowest MMSE value from 25 to 26 and decreasing the highest MMSE value from 30 to 29. This resulted in the inclusion of 19 aMCI and 13 MCI PD patients and in a non-significant difference on the MMSE. Subsequently, AD patients were compared to PDD patients. Finally, aMCI patients were compared to AD patients and MCI PD to PDD patients to look for disease-specific differences in the pattern of cognitive deficits.

\section{Results}

Age, gender and average scores on each of the tasks for the different groups of participants are summarized in tables 1 and 2. Notably, the two groups with MCI (aMCI vs. PD) and with dementia (AD vs. PDD) did not differ in age, education and MMSE score.

\section{Differences between Diseases}

After matching for disease severity by adapting criteria for the MMSE scores (see above), aMCI and MCI PD patients differed significantly on attention tests (ZVT: $p=0.021$, Digit Span forward: $p=0.028$ ), episodic memory (word list delayed recall: $p=0.02$, word list savings: $p=$ 0.018 , word list recognition commission errors: $p=0.014$ ), and visuoconstruction (figure 


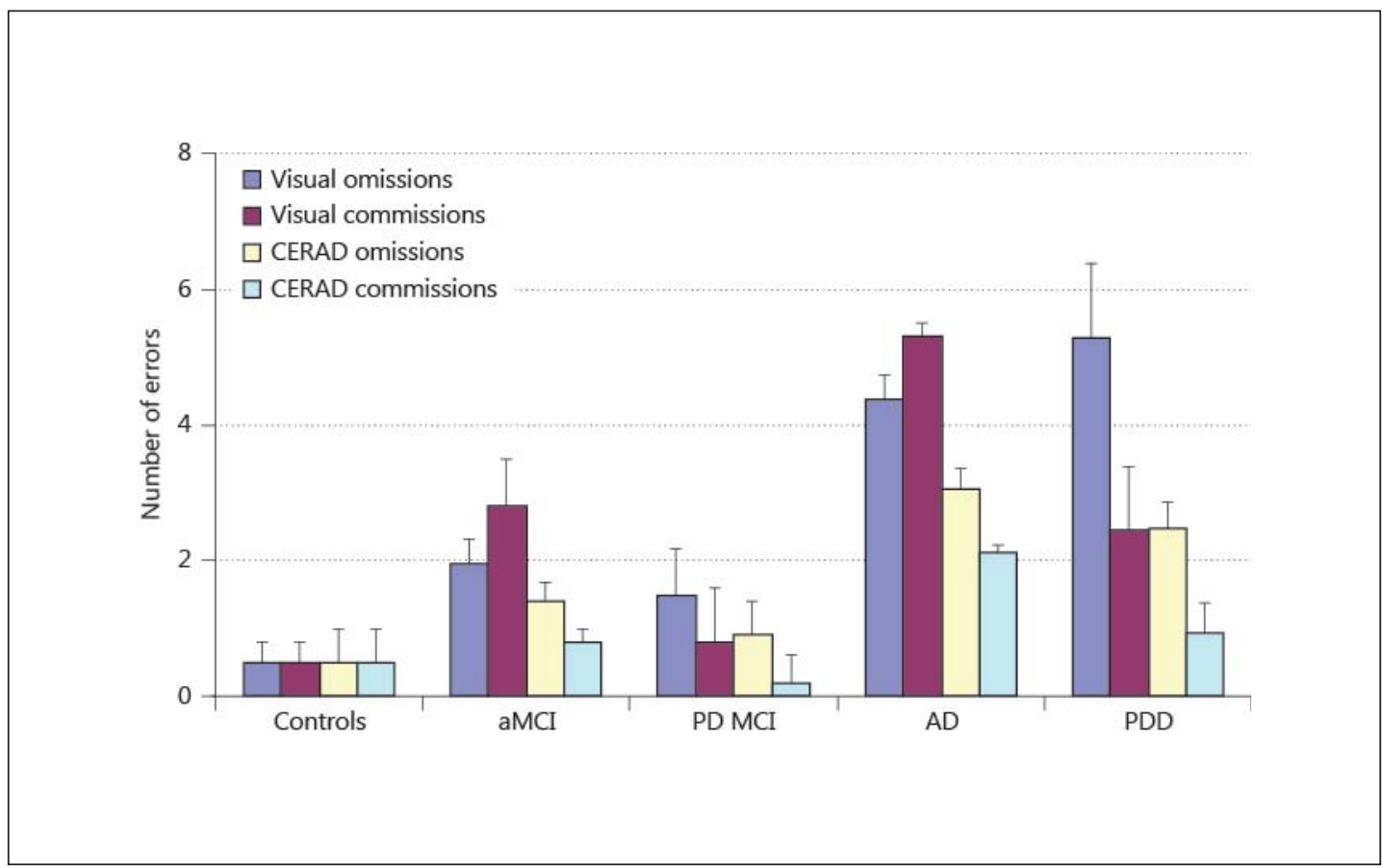

Fig. 1. Only aMCI and $\mathrm{AD}$ patients performed more commissions than omissions in the visual recognition task. Moreover, aMCI patients differed from PD MCI patients in CERAD word list commissions, and patients with AD differed from patients with PDD.

copying: $\mathrm{p}=0.041$ ). MCI PD patients were slower than aMCI patients and solved less visuoconstructional tasks, but reproduced more digits and words. The same analyses for the entire group of patients gave the same pattern of results.

AD and PDD patients only differed significantly in commission errors on the experimental visual recognition task $(p=0.027)$, in commission errors on the word list recognition task $(p=0.049)$ and in depressive mood $(p=0.024)$, but not in visuoconstruction and attention. AD patients made more errors but showed less depressive mood than PDD patients.

Taken together, the results show that PD patients, both in the early and late phase, differed from patients with aMCI and probable AD in commission errors, but not in omissions.

\section{Differences in Severity}

For both disorders, the groups with dementia showed a more severe and broader impairment with respect to language and memory functions than patients in the early phase suffering from MCI (see columns 1 and 3 of table 2). AD patients differed significantly from aMCI patients in attention (ZVT, $\mathrm{p}=0.001$ ), but PD patients did not. The same applies for visuoconstruction $(\mathrm{p}=0.015)$. PDD patients differed from MCI PD patients in depressive $\operatorname{mood}(\mathrm{p}=0.018)$, but aMCI and $\mathrm{AD}$ patients did not differ with respect to depression.

Interestingly, both groups with dementia performed worse compared to their MCI groups in recognition performance (discriminability index of the CERAD word list learning: AD patients: $p<0.001$; PD patients: $p=0.009$ ). Apparently, they do so for different reasons: whereas $\mathrm{AD}$ patients, compared to aMCI patients, showed a significant increase in omissions (visual recognition task: $\mathrm{p}=0.002$; word list recognition task: $\mathrm{p}=0.015$ ) and in commissions (visual recognition task: $\mathrm{p}=0.015$; word list recognition task: $\mathrm{p}=0.019$ ) on both recognition 
Table 3. Significant differences between groups according to disease etiology and disease stage

\begin{tabular}{|c|c|c|}
\hline & Significant differences in & \\
\hline$a M C I$ & $\begin{array}{l}\text { ZVT, DS forward, word list delayed recall, } \\
\text { word list savings, word list recognition } \\
\text { commission errors, copying }\end{array}$ & $M C I P D$ \\
\hline $\begin{array}{l}\text { ZVT, DS backward, naming, FAS, semantic } \\
\text { fluency, verbal comprehension, omissions } \\
\text { and commissions in visual recognition, } \\
\text { word list learning, delayed recall, savings, } \\
\text { omissions and commissions in word list } \\
\text { recognition, copying, visuoconstructive } \\
\text { delayed recall and savings }\end{array}$ & $\downarrow$ & $\begin{array}{l}\text { BDI, DS forward and backward, naming, } \\
\text { semantic fluency, verbal comprehension, } \\
\text { omissions in visual recognition, word list } \\
\text { learning, delayed recall, savings, } \\
\text { omissions in word list recognition, } \\
\text { visuoconstructive delayed recall and } \\
\text { savings }\end{array}$ \\
\hline$A D$ & $\begin{array}{l}B D I \text {, word list learning, word list } \\
\text { commission errors, visual recognition } \\
\text { commissions }\end{array}$ & $P D D$ \\
\hline
\end{tabular}

BDI = Beck Depression Inventory; DS = Digit Span; FAS = phonological word fluency test.

tasks, patients with PDD showed a significant increase only for omissions on both recognition tasks (visual recognition task: $p=0.001$; word list recognition task: $p=0.003$ ) (fig. 1).

A comprehensive overview of significant differences between the four groups is presented in table 3.

\section{Discussion}

As observed in earlier investigations [15, 33], MCI PD patients suffered from attentional and visuoconstructive deficits, whereas episodic memory impairments were detected in patients with aMCI. However, in an advanced stage of the two diseases, the deficits in attentional or language functions and visuoconstruction were similar. In contrast, depressive mood was a distinctive feature in PDD, but was not increased in AD. AD and PDD patients differed in learning (not in delayed recall), and AD patients made more commission errors than PDD patients.

In advanced $\mathrm{AD}$ and PDD, similar patterns of cognitive deficits were observed, particularly involving language functions, attention and visuoconstructive functions. These cognitive domains rely largely on temporal and parietal areas: visual attention and visuoconstructive performance are related to the integrity of the right parietal lobe, whereas verbal working memory (Digit Span, which is often classified as a test of attention) and verbal fluency are associated with the left parietal and temporal areas [34]. It is well known that, in neuropathological terms, AD starts in the mediotemporal lobe, but during the Braak stage $\mathrm{V}$ it spreads to the temporoparietal areas. Enlarged sulci [35, 36] and hypoperfusion of these areas [37] are hallmarks of AD progression. For PD, impairments in visuoconstruction and attention belong to the defining and early features of PDD and seem to be due to cognitive slowing (visual attention and verbal working memory) and impaired executive functions [10, 12, 33, 38]. According to our findings, the deficits worsen somewhat over the course of the disease, as documented by significant decrements on tests for these functions between MCI PD and PDD patients, but this progress seems to be smaller than that in AD. Consequently, the patterns of deficit seem to lose their specificity during the disease course. 
The two groups with MCI differed in delayed recall and savings of word list learning and in commission errors, but not in omissions on the recognition tasks. From a cognitive point of view, the omissions and commissions in recognition can be linked to two distinct processes. According to the dual process theory of recognition [39], items may be remembered either by recollection or by familiarity. A deficit in recollection may lead to an increase of false-positive responses because recognition is then primarily based on the feeling of familiarity, in particular for related items. However, a deficit in familiarity processing might lead to less hits and more omissions, but not to more commission. Such dissociation was exactly what we found. Several previous studies [40-44] have shown that recognition in AD patients is strongly influenced by item familiarity because of the fundamental deficit in episodic memory. Similar results for increased intrusions in $\mathrm{AD}$ patients, compared to patients with other kinds of dementia, have been shown with clinical tests $[45,46]$ and with the visual recognition task described in the Methods section [32].

Research on recognition deficits in PD patients has revealed mixed results. Recently, Cohn et al. [47] have shown that memory performance of PD patients is strongly influenced by the nature of the encoding process: after shallow encoding, PD patients suffer from a familiarity deficit and following deep encoding, they have a recollection deficit. Our two recognition tasks both required visual perception (identifying objects and reading words). In the object recognition task, we did not inform the patients of the subsequent recognition task, and in the word list task, we used a set of unrelated words without any structuring cues. One may argue that these task characteristics do not stimulate deep encoding, and this may explain why PD patients suffered from a specific familiarity deficit on both recognition tasks.

PDD patients performed better than AD patients in learning and recognition, while MCI PD patients scored better than aMCI patients on the recognition and delayed free recall tasks. First, recognition is the easiest form of consciously remembering past experiences, and impairment in recognition can be considered as impairment in consolidation rather than as impairment in delayed free recall. Second, delayed free recall relies also on executive functions (strategic search processes), and this effect becomes more pronounced after a delay. Hence, we would argue that the difference in delayed free recall between MCI PD and aMCI patients disappeared in the later stage, not due to the development of a similar deficit in episodic memory, but due to an increasing deficit in strategic search. As this deficit is limited in MCI PD patients, it only affected performance in the more difficult delayed free recall task of word list learning and not in word list learning. Due to the higher deficit severity in PDD, it also affected word list learning and impaired the performance in the delayed free recall task to the same degree as the episodic memory deficit of the AD patients.

This finding of a persistent difference in recognition memory between AD and PDD patients fits well with the progressive neuropathological characteristics of the two diseases: AD starts in the entorhinal cortex and the hippocampus and thus impairs recollection, whereas in PD the main pathological feature is a decrease in the dopaminergic modulation of the frontal cortex [17]. As previous studies show, in addition to other structures, the frontal cortex is significant for the feeling of familiarity [48].

The frontal cortex might also be involved in the increased incidence of depression as observed in PDD. In the beginning, the loss of dopaminergic neurons mainly occurs in the dorsal striatum, but in the course of the disease, it spreads to more ventral parts of the striatum. The ventral part of the striatum is an essential component of the basal ganglia loops, which instantiate cognitive and affective functions. Consequently, the decrease in familiarity and mood may be due to the progression of the neurodegenerative process spreading to the ventral striatum. A correlation between PD progression, depression and dementia has been shown by several studies $[3,4,49,50]$. 
Certain limitations of the present study need to be acknowledged. First, we used a crosssectional study design, but a longitudinal design would have been more appropriate. This is especially true for aMCI patients since we have to assume that only a percentage of them will convert into AD patients. Second, the AD patients have been recruited from an inpatient setting, which may have introduced a selection bias. On the contrary, the diagnosis of AD has been based on a clinical investigation, a neuropsychological assessment and on CSF markers, which should have helped to avoid any major influence in the diagnosis. Third, the number of patients per group was low, especially in the PDD patient group.

In summary, our results indicate that from an early state of MCI to the state of dementia there is a common loss of functions in AD and PD, mainly concerning parietal and temporal functions such as language and attention. In contrast, other cognitive and affective deficits remain specific for each of the two disorders, and these deficits concern recollection as mediotemporal function and mood as basal ganglia function.

Therefore, with respect to clinical neuropsychological assessment, our results argue for a differentiated evaluation of omissions and commissions in recognition tasks, apart from a combined evaluation of discrimination measures such as the discriminability index of the CERAD-NP battery. At least in this investigation, the differences between AD and PD patients were maximal for commission errors related to recollection and not for omission errors related to familiarity. For clinical purposes, this investigation also argues that it is important to check the diagnosis carefully in the case that probable AD patients show relevant depressive symptoms, since this was rarely the case in our patient groups.

\section{Disclosure Statement}

The authors confirm that there are no conflicts of interest, financial interests and patent holdings related to this article. The authors also received no financial report related to the research documented in this article.

\section{References}

1 Aarsland D, Andersen K, Larsen JP, Lolk A, Nielsen H, Kragh-Sorensen P: Risk of dementia in Parkinson's disease: a community-based, prospective study. Neurology 2001;56:730-736.

$\checkmark 2$ Aarsland D, Tandberg E, Larsen JP, Cummings JL: Frequency of dementia in Parkinson disease. Arch Neurol 1996;53:538-542.

3 Riedel O, Klotsche J, Spottke A, Deuschl G, Forstl H, Henn F, et al: Frequency of dementia, depression, and other neuropsychiatric symptoms in 1,449 outpatients with Parkinson's disease. J Neurol 2010;257:1073-1082.

4 Riedel O, Klotsche J, Spottke A, Deuschl G, Forstl H, Henn F, et al: Cognitive impairment in 873 patients with idiopathic Parkinson's disease. Results from the German Study on Epidemiology of Parkinson's Disease with Dementia (GEPAD). J Neurol 2008;255:255-264.

5 Williams-Gray CH, Foltynie T, Brayne CE, Robbins TW, Barker RA: Evolution of cognitive dysfunction in an incident Parkinson's disease cohort. Brain 2007;130:1787-1798.

6 Cahn-Weiner DA, Grace J, Ott BR, Fernandez HH, Friedman JH: Cognitive and behavioral features discriminate between Alzheimer's and Parkinson's disease. Neuropsychiatry Neuropsychol Behav Neurol 2002;15:79-87.

7 Galvin JE, Pollack J, Morris JC: Clinical phenotype of Parkinson disease dementia. Neurology 2006;67:16051611.

-8 Pagonabarraga J, Kulisevsky J, Llebaria G, Garcia-Sanchez C, Pascual-Sedano B, Gironell A: Parkinson's diseasecognitive rating scale: a new cognitive scale specific for Parkinson's disease. Mov Disord 2008;23:998-1005.

-9 Saka E, Elibol B: Enhanced cued recall and clock drawing test performances differ in Parkinson's and Alzheimer's disease-related cognitive dysfunction. Parkinsonism Relat Disord 2009;15:688-691.

10 Song IU, Kim JS, Jeong DS, Song HJ, Lee KS: Early neuropsychological detection and the characteristics of Parkinson's disease associated with mild dementia. Parkinsonism Relat Disord 2008;14:558-562.

11 Goetz CG, Emre M, Dubois B: Parkinson's disease dementia: definitions, guidelines, and research perspectives in diagnosis. Ann Neurol 2008;64(suppl 2):S81-S92. 
12 Woods SP, Troster AI: Prodromal frontal/executive dysfunction predicts incident dementia in Parkinson's disease. J Int Neuropsychol Soc 2003;9:17-24.

-13 Janvin CC, Larsen JP, Aarsland D, Hugdahl K: Subtypes of mild cognitive impairment in Parkinson's disease: progression to dementia. Mov Disord 2006;21:1343-1349.

14 Janvin CC, Larsen JP, Salmon DP, Galasko D, Hugdahl K, Aarsland D: Cognitive profiles of individual patients with Parkinson's disease and dementia: comparison with dementia with Lewy bodies and Alzheimer's disease. Mov Disord 2006;21:337-342.

15 Metzler-Baddeley C: A review of cognitive impairments in dementia with Lewy bodies relative to Alzheimer's disease and Parkinson's disease with dementia. Cortex 2007;43:583-600.

16 Oh ES, Lee JH, Jeong SH, Sohn EH, Lee AY: Comparisons of cognitive deterioration rates by dementia subtype. Arch Gerontol Geriatr 2011;53:320-322.

17 Braak H, Braak E, Yilmazer D, de Vos RA, Jansen EN, Bohl J: Pattern of brain destruction in Parkinson's and Alzheimer's diseases. J Neural Transm 1996;103:455-490.

18 Jellinger KA, Seppi K, Wenning GK, Poewe W: Impact of coexistent Alzheimer pathology on the natural history of Parkinson's disease. J Neural Transm 2002;109:329-339.

19 Mastaglia FL, Johnsen RD, Byrnes ML, Kakulas BA: Prevalence of amyloid-beta deposition in the cerebral cortex in Parkinson's disease. Mov Disord 2003;18:81-86.

20 Clinton LK, Blurton-Jones M, Myczek K, Trojanowski JQ, LaFerla FM: Synergistic interactions between Abeta, tau, and alpha-synuclein: acceleration of neuropathology and cognitive decline. J Neurosci 2010;30:72817289.

21 Sabbagh MN, Adler CH, Lahti TJ, Connor DJ, Vedders L, Peterson LK, et al: Parkinson disease with dementia: comparing patients with and without Alzheimer pathology. Alzheimer Dis Assoc Disord 2009;23:295-297.

-22 Haldenwanger A, Eling P, Kastrup A, Hildebrandt H: Correlation between cognitive impairment and CSF biomarkers in amnesic MCI, non-amnesic MCI, and Alzheimer's disease. J Alzheimers Dis 2010;22:971-980.

23 Hildebrandt H, Haldenwanger A, Eling P: False recognition correlates with amyloid-beta (1-42) but not with total tau in cerebrospinal fluid of patients with dementia and mild cognitive impairment. J Alzheimers Dis 2009;16:157-165.

24 Bremen ML: Laborinfo Demenz, 2004 www.mlhb.de/fileadmin/user_upload/Fachinfo/Laborinfo/ Demenz_270105.pdf.

Hoehn MM, Yahr MD: Parkinsonism: onset, progression and mortality. Neurology 1967;17:427-442.

26 Morris JC, Heyman A, Mohs RC, Hughes JP, van Belle G, Fillenbaum G, et al: The Consortium to Establish a Registry for Alzheimer's Disease (CERAD). Part I. Clinical and neuropsychological assessment of Alzheimer's disease. Neurology 1989;39:1159-1165.

27 Horn W: Leistungsprüfsystem (LPS). Bern, Hans Huber Verlag, 1983.

28 Oswald WD, Fleischmann UM: Das Nürnberger Altersinventar (NAI). Göttingen, Hogrefe, 1997.

29 Wechsler D: Wechsler Memory Scale-Revised Manual, San Antonio, TX, Psychological Corporation, 1987.

30 Lehrl S, Merz J, Burkhard G, et al: Mehrfach-Wortschatz-Intelligenztest (MWT-A). Erlangen, Perimed, 1991.

31 Beck AT, Hautzinger M, Bailer M, Worall H, Keller F.: BDI-Beck-Depressions-Inventar, ed 2. Göttingen, Hogrefe, 1985.

-32 Hildebrandt H, Haldenwanger A, Eling P: False recognition helps to distinguish patients with Alzheimer's disease and amnestic MCI from patients with other kinds of dementia. Dement Geriatr Cogn Disord 2009;28: 159-167.

-33 Litvan I, Aarsland D, Adler CH, Goldman JG, Kulisevsky J, Mollenhauer B, et al: MDS Task Force on mild cognitive impairment in Parkinson's disease: critical review of PD-MCI. Mov Disord 2011;26:1814-1824.

34 Kolb B, Wishaw IQ: Fundamentals of Human Neuropsychology, ed 6. New York, NY, Worth Publishers, 2009.

-35 Melzer TR, Watts R, MacAskill MR, Pitcher TL, Livingston L, Keenan RJ, et al: Grey matter atrophy in cognitively impaired Parkinson's disease. J Neurol Neurosurg Psychiatry 2012;83:188-194.

36 Song SK, Lee JE, Park HJ, Sohn YH, Lee JD, Lee PH: The pattern of cortical atrophy in patients with Parkinson's disease according to cognitive status. Mov Disord 2011;26:289-296.

-37 Turjanski N, Brooks DJ: PET and the investigation of dementia in the parkinsonian patient. J Neural Transm Suppl 1997;51:37-48.

-38 Petrova M, Raycheva M, Zhelev Y, Traykov L: Executive functions deficit in Parkinson's disease with amnestic mild cognitive impairment. Am J Alzheimers Dis Other Demen 2010;25:455-460.

-39 Yonelinas AP: Receiver-operating characteristics in recognition memory: evidence for a dual-process model. J Exp Psychol Learn Mem Cogn 1994;20:1341-1354.

40 Abe N, Fujii T, Nishio Y, Iizuka 0, Kanno S, Kikuchi H, et al: False item recognition in patients with Alzheimer's disease. Neuropsychologia 2011;49:1897-1902.

-41 Budson AE, Michalska KJ, Sullivan AL, Rentz DM, Daffner KR, Schacter DL: False recognition in Alzheimer disease: evidence from categorized pictures. Cogn Behav Neurol 2003;16:16-27.

42 Gainotti G, Marra C, Villa G, Parlato V, Chiarotti F: Sensitivity and specificity of some neuropsychological markers of Alzheimer dementia. Alzheimer Dis Assoc Disord 1998;12:152-162.

43 Gallo DA, Shahid KR, Olson MA, Solomon TM, Schacter DL, Budson AE: Overdependence on degraded gist memory in Alzheimer's disease. Neuropsychology 2006;20:625-632. 
44 Gold CA, Marchant NL, Koutstaal W, Schacter DL, Budson AE: Conceptual fluency at test shifts recognition response bias in Alzheimer's disease: implications for increased false recognition. Neuropsychologia 2007;45: 2791-2801.

45 Davis KL, Price CC, Kaplan E, Libon DJ: Error analysis of the nine-word California Verbal Learning Test (CVLT-9) among older adults with and without dementia. Clin Neuropsychol 2002;16:81-89.

-46 Fine EM, Delis DC, Wetter SR, Jacobson MW, Hamilton JM, Peavy G, et al: Identifying the 'source' of recognition memory deficits in patients with Huntington's disease or Alzheimer's disease: evidence from the CVLT-II. J Clin Exp Neuropsychol 2008;30:463-470.

47 Cohn M, Moscovitch M, Davidson PS: Double dissociation between familiarity and recollection in Parkinson's disease as a function of encoding tasks. Neuropsychologia 2010;48:4142-4147.

48 MacPherson SE, Bozzali M, Cipolotti L, Dolan RJ, Rees JH, Shallice T: Effect of frontal lobe lesions on the recollection and familiarity components of recognition memory. Neuropsychologia 2008;46:3124-3132.

-49 Santangelo G, Vitale C, Trojano L, Longo K, Cozzolino A, Grossi D, et al: Relationship between depression and cognitive dysfunctions in Parkinson's disease without dementia. J Neurol 2009;256:632-638.

-50 Troster AI, Paolo AM, Lyons KE, Glatt SL, Hubble JP, Koller WC: The influence of depression on cognition in Parkinson's disease: a pattern of impairment distinguishable from Alzheimer's disease. Neurology 1995;45: 672-676. 